\title{
SYMMETRY BREAKING FOR A CLASS OF SEMILINEAR ELLIPTIC PROBLEMS
}

\author{
MYTHILY RAMASWAMY AND P. N. SRIKANTH
}

\begin{abstract}
We study positive solutions of the Dirichlet problem for $-\Delta u=$ $u^{p}-\lambda, p>1, \lambda>0$, on the unit ball $\Omega$. We show that there exists a positive solution $\left(u_{0}, \lambda_{0}\right)$ of this problem which satisfies in addition $\partial u_{0} / \partial n=0$ on $\partial \Omega$. We prove also that at $\left(u_{0}, \lambda_{0}\right)$, the symmetry breaks, i.e. asymmetric solutions bifurcate from the positive radial solutions.
\end{abstract}

1. Introduction. We consider here the symmetry breaking for semilinear elliptic problems of the type

$$
\begin{aligned}
-\Delta u & =f(u, \lambda) \quad \text { in } B_{1}, \\
u & =0 \quad \text { on } \partial B_{1},
\end{aligned}
$$

where $B_{1}$ is the unit ball on $\mathbf{R}^{n}, n \geq 2$, and $f: \mathbf{R} \times \mathbf{R} \rightarrow \mathbf{R}$ is a $C^{2}$ function. We say that the symmetry breaks at $\lambda$ if the radial solution $\left(u_{0}, \lambda_{0}\right)$ is a nonradial bifurcation point, i.e. if every neighborhood of $\left(u_{0}, \lambda_{0}\right)$ contains solutions of (1.1), $(u, \lambda)$ with $u$ nonradial.

Symmetry breaking problems for positive solutions of semilinear elliptic equations have been studied by Smoller and Wasserman $[\mathbf{4}, \mathbf{5}]$. In fact they prove that for the function $f \in C^{2}$ satisfying

$$
\begin{aligned}
f(0) & <0, \quad\left(\frac{f(t)}{t}\right)^{\prime}<0, \\
f^{\prime \prime}(t) & \leq 0 \quad \forall t>0 \quad \text { and } \quad f(t)>0 \quad \text { for some } t>0,
\end{aligned}
$$

there is an interval $\left(R_{1}, R_{2}\right]$ such that for all $R \in\left(R_{1}, R_{2}\right]$ there exists a unique positive radial solution of

$$
\begin{aligned}
-\Delta u & =f(u) \quad \text { in } B_{R} \subset \mathbf{R}^{n}, \\
u & =0 \quad \text { on } \partial B_{R}
\end{aligned}
$$

and only when $R=R_{2}$, the necessary condition for symmetry breaking, namely

$$
\partial u / \partial n=0 \quad \text { on } \partial B_{R},
$$

is satisfied. They conjecture that the symmetry breaks at $R_{2}$. In both [5] and [1], it is shown that there exists a nonempty subclass of functions satisfying (1.2), for which this conjecture is true. However only for a "generic" subclass, the result is true, mainly due to the difficulty involved in verifying a certain nondegeneracy. Here we give an explicit class of functions depending on a parameter $\lambda$, namely

$$
f(u, \lambda)=u^{p}-\lambda, \quad p>1,
$$

Received by the editors November 5,1986 .

1980 Mathematics Subject Classification (1985 Revision). Primary 35J25; Secondary 35P30. 
for which the symmetry breaks when we consider the problem in the unit ball. However this function does not satisfy (1.2).

The one dimensional problem for the odd function $f(u, \lambda)=u|u|^{p-1}-\lambda$ was studied in [3]. The behavior of the solution branches described there leads to the conjecture that for the higher dimensional analogue of the problem, when a positive solution satisfies $\partial u / \partial n=0$ on $\partial \Omega$, symmetry breaks at that point. We prove here that it does happen indeed.

2. Main results. Consider the equation

$$
\begin{aligned}
-\Delta u & =u^{p}-\lambda \quad \text { in } \Omega \equiv B_{1} \subset \mathbf{R}^{n}, \\
u & =0 \quad \text { on } \partial \Omega .
\end{aligned}
$$

Here $p<(n+2) /(n-2)$ in view of Pohazaev's nonexistence result. From [2], we know that the positive solutions of $(2.1)$ are radial. At $\lambda=0$, the unique positive solution $u_{0}$ of $(2.1)$ is in fact nondegenerate. Since a proof of this nondegeneracy could not be located in the literature, we indicate the proof here for the sake of completeness. Now onwards, we let

$$
f(u)=u^{p}, \quad 1<p<(n+2) /(n-2) .
$$

Lemma 1 will show that $\left(u_{0}, 0\right)$ is radially nondegenerate. We just remark here that nonradial degeneracy can also be ruled out at $\left(u_{0}, 0\right)$ and in fact all along the positive solution branch with $\partial u / \partial n<0$ on $\partial \Omega$.

LEMMA 1. Let $u$ be a positive solution of (2.1) with $\partial u / \partial n \leq 0$ on $\partial \Omega$. Assume that there exists a radial eigenfunction $v$ satisfying

$$
\begin{aligned}
-\Delta v & =f^{\prime}(u) v \quad \text { in } \Omega, \\
v & =0 \quad \text { on } \quad \partial \Omega .
\end{aligned}
$$

Then

$$
v^{\prime}(1) u^{\prime}(1)=-\frac{2 p \lambda}{p-1} \int_{0}^{1} r^{n-1} v(r) d r .
$$

PROOF. It is easy to verify

$$
-\Delta\left(r u^{\prime}\right)=f^{\prime}(u)\left(r u^{\prime}\right)+2(f(u)-\lambda) \quad \text { in } \Omega .
$$

Now using the equation for $u$, we have

$$
\begin{aligned}
-\Delta\left(r u^{\prime}+\frac{2}{p-1} u\right) & =f^{\prime}(u)\left(r u^{\prime}+\frac{2}{p-1} u\right)-\frac{2 p}{p-1} \lambda \quad \text { in } \Omega \\
\left(r u^{\prime}+\frac{2}{p-1} u\right) & =u^{\prime}(1) \quad \text { on } \partial \Omega .
\end{aligned}
$$

We multiply $(2.2)$ by $\left(r u^{\prime}+2 u /(p-1)\right)$ and $(2.4)$ by $v$, subtract one from the other and integrate over $\Omega$. then (2.3) follows.

At $\left(u_{0}, 0\right)$, if there were any radial degeneracy, then Lemma 1 yields an absurd equation $u^{\prime}(1) v^{\prime}(1)=0$. Thus $\left(u_{0}, 0\right)$ has to be nondegenerate. Now the implicit function theorem gives the branch of positive solutions of (2.1) for $\lambda$ close to 0 .

We study the behavior of this branch for large positive $\lambda$. The following theorem is an extension of the nonexistence result in one dimension. (Cf. [3].) 
THEOREM 1. There exists no positive solution to equation (2.1) for large positive $\lambda$.

PROOF. The proof follows by contradiction. Let if possible $\left(u_{j}, \lambda_{j}\right)$ be a sequence of positive solutions, with $\lambda_{j} \rightarrow \infty$. Since $u_{j}$ is radial, it satisfies

$$
\begin{gathered}
-u_{j}^{\prime \prime}-(n-1) u_{j}^{\prime} / r=f\left(u_{j}\right)-\lambda_{j} \quad \text { in }(0,1), \\
u_{j}^{\prime}(0)=0 ; \quad u_{j}(1)=0 .
\end{gathered}
$$

Now $\Delta u_{j}<0$ at 0 , it being the maximum. At $1, \Delta u_{j}>0$. Thus there exists a point $a$ where $f\left(u_{j}(a)\right)-\lambda_{j}=0$. Now choose a point $b \in(a, 1)$ such that

$$
f\left(u_{j}(b)\right)=\lambda_{j} / 2 \text {. }
$$

We first prove that $b \rightarrow 1$ and hence $a$ also, for $\lambda_{j} \rightarrow \infty$. We have in $[b, 1]$,

$$
\frac{\lambda_{j}}{2}<\frac{1}{r^{n-1}}\left(\frac{d}{d r}\left(r^{n-1} u_{j}^{\prime}\right)\right) .
$$

Integration of this from $b$ to 1 leads to

$$
\frac{\lambda_{j}}{2 n}\left(1-b^{n}\right)<u_{j}^{\prime}(1)-b^{n-1} u_{j}^{\prime}(b)=b^{n-1}\left|u_{j}^{\prime}(b)\right|-\left|u_{j}^{\prime}(1)\right| .
$$

Multiplying (2.5) by $u_{j}^{\prime} r^{2(n-1)}$ and integrating between $b$ and 1 ,

$$
\begin{aligned}
& \frac{1}{2}\left[u_{j}^{\prime 2}(b) b^{2(n-1)}-u_{j}^{\prime 2}(1)\right] \\
& \quad=-2(n-1) \int_{b}^{1}\left(F\left(u_{j}\right)-\lambda u_{j}\right) r^{2 n-3}+\left[\left(F\left(u_{j}\right)-\lambda u\right) r^{2 n-2}\right]_{b}^{1} \\
& \quad \leq 2(n-1) \int_{b}^{1}\left(\lambda u_{j}-F\left(u_{j}\right)\right) r^{2 n-3}+\left(2-\frac{1}{p+1}\right)\left(u_{j}(b)\right)^{p+1} b^{2 n-2} \\
& \quad \leq\left(u_{j}(b)^{p+1}\left(2(n-1)+2-\frac{1}{p+1}\right)\right) .
\end{aligned}
$$

Here $F(u)$ stands for the primitive of $f$, namely $\left(u^{p+1}\right) /(p+1)$. Further we have

$$
\begin{aligned}
\left|u_{j}^{\prime}(b)\right| b^{n-1}-\left|u_{j}^{\prime}(1)\right| & \leq \sqrt{u_{j}^{\prime}(b) b^{2(n-1)}-u_{j}^{\prime 2}(1)} \\
& \leq c\left|u_{j}(b)\right|^{(p+1) / 2}
\end{aligned}
$$

This along with (2.7) results in

$$
\left(1-b^{n}\right)<c_{1}\left|u_{j}(b)\right|^{((p+1) / 2)-p} .
$$

As $p>1$, this goes to 0 when $\lambda_{j} \rightarrow \infty$, in view of (2.6). Thus $b \rightarrow 1$.

The functions $f$ and $u_{j}$ being convex and $f$ being monotone, the composition $f \circ u_{j}$ is convex in $[a, 1]$. Thus

$$
\begin{gathered}
f \circ u_{j}(1 / 2+a / 2) \leq\left(f \circ u_{j}(a)\right) / 2=f \circ u_{j}(b), \\
\Rightarrow(1+a) / 2>b ; \quad(1-b)>(b-a) .
\end{gathered}
$$

Hence it follows that for $\lambda_{j}$ large enough, $1-a<\varepsilon$, for a preassigned $\varepsilon$. Let $\mu_{1}=\mu_{1}\left(-\Delta, B_{1-\varepsilon}\right)$ be the first eigenvalue of $-\Delta$ in $B_{1-\varepsilon}$, with Dirichlet boundary conditions. Define

$$
v(x)=u_{j}(x)-u_{j}(a) .
$$


Choose $\lambda_{j}$ large enough so that $f\left(u_{j}(a)\right) / u_{j}(a)>\mu_{1}$. From the equation, we get

$$
\begin{aligned}
-\Delta v & >\mu_{1}\left(-\Delta, B_{a}\right) v \quad \text { in } B_{a} \\
v & \geq 0 \quad \text { in } B_{a} \\
v & =0 \quad \text { on } \partial B_{a} .
\end{aligned}
$$

This is easily shown to be impossible by comparing this with the eigenvalue equation in $B_{a}$. Thus the theorem holds.

Now it follows that the positive solution branch does not extend beyond a certain $\lambda_{0}$. It has to encounter a singular point. Theorem 2 shows that this cannot be a radial singularity.

THEOREM 2. There is no radial degeneracy all along the positive solution branch satisfying (2.1) with $\partial u / \partial n \leq 0$ on $\partial \Omega$.

ProOF. Assume if possible, for some positive solution $(u, \lambda)$ of $(2.1)$, there exists a radial eigenfunction $v$ satisfying (2.2). The idea is to get a contradiction by comparing the equation for a suitable test function with that of $v$. Define

$$
w(r)=\int_{0}^{r} t^{n-1} v d t
$$

It is easy to verify

$$
-\Delta w=-r^{n-1} v^{\prime}-2(n-1) r^{n-2} v \quad \text { in } \Omega .
$$

Now multiplying $(2.8)$ by $v$ and $(2.2)$ by $w$, integrating and subtracting one from the other,

$$
v^{\prime}(1) w(1)=-\int_{0}^{1} r^{2(n-1)} v v^{\prime}-2(n-1) \int_{0}^{1} r^{2 n-3} v^{2}-\int_{0}^{1} f^{\prime}(u) v w r^{n-1} .
$$

After an integration by parts in the first and last terms, this leads to

$$
v^{\prime}(1) w(1)=-(n-1) \int_{0}^{1} r^{2 n-3} v^{2}+\frac{1}{2} \int_{0}^{1} f^{\prime \prime}(u) u^{\prime} w^{2}
$$

Since $u>0$ and $u^{\prime}<0$ in $(0,1)$, the right-hand side is completely negative. $v$ can always be chosen so as to have $v^{\prime}(1)>0$. Then it follows that

$$
\int_{0}^{1} r^{n-1} v<0
$$

Now Lemma 1 gives

$$
-((p-1) / 2 p \lambda) v^{\prime}(1) u^{\prime}(1)<0 .
$$

This leads to a contradiction as $u^{\prime}(1) \leq 0$. Thus the positive solution branch for (2.1) is radially nondegenerate.

From the above two theorems, we conclude that there exists a positive solution $\left(u_{0}, \lambda_{0}\right)$ for $(2.1)$ satisfying $\partial u_{0} / \partial n=0$ on $\partial \Omega$. This is indeed a singular point since the linearized problem has at least $n$ nonradial solutions $\left\{\partial u / \partial x_{i}\right\}_{1 \leq i \leq n}$. Lemma 2.2 of [1] (also cf. Theorem 20 of [4]) shows that there can be $n$ nonradial solutions or $(n+1)$ solutions including one radial solution. Theorem 2 rules out the second possibility. Now to show the symmetry breaking at $\left(u_{0}, \lambda_{0}\right)$, we need the following definitions and Theorem 3. 
We define

$$
G(u, \lambda) \equiv-\Delta u-f(u)+\lambda
$$

from the space

$$
X=\left\{u \in C_{0}^{2, \alpha}: u\left(x_{1}, \ldots, x_{n-1}, x_{n}\right)=u\left(-x_{1},-x_{2}, \ldots,-x_{n-1}, x_{n}\right)\right\}
$$

into $C^{\alpha}(\Omega)$. We have

$$
\begin{aligned}
& \operatorname{ker} G_{u}\left(u_{0}, \lambda_{0}\right)=\operatorname{span}\left\{\partial u_{0} / \partial x_{n}\right\} \\
& \operatorname{dimension}\left(\operatorname{ker} G_{u}^{0}\right)=\operatorname{codimension}\left(R a G_{u}^{0}\right)=1
\end{aligned}
$$

Let $\phi$ be the unique solution of the problem

$$
G_{u}^{0} \phi+G_{\lambda}^{0}=0 ; \quad \int_{\Omega} \phi \frac{\partial u_{0}}{\partial x_{n}}=0
$$

Note that $G_{\lambda}^{0}=1$ is in $R a\left(G_{u}^{0}\right)$ since

$$
\int_{\Omega} 1 \cdot \frac{\partial u_{0}}{\partial x_{n}}=0
$$

Now we prove the symmetry breaking.

THEOREM 3. $\left(u_{0}, \lambda_{0}\right)$ is a nonradial bifurcation point.

PROOF. We use standard arguments to derive the bifurcation equation. This extends the proof for one dimension as in Proposition (5.1) in [3]. Assuming that the branch is given by $(u(s), \lambda(s))$, we have

$$
G(u(s), \lambda(s))=0 \quad \text { for } s \in\left(s_{0}-\varepsilon, s_{0}+\varepsilon\right)
$$

where $\left(u\left(s_{0}\right), \lambda\left(s_{0}\right)\right)=\left(u_{0}, \lambda_{0}\right)$. Differentiation with respect to $s$ gives $G_{u}^{0} u^{\prime}(s)+$ $G_{\lambda}^{0} \lambda^{\prime}(s)=0$. This along with (2.12) yields,

$$
u^{\prime}(s)-\lambda^{\prime}(s) \phi=\alpha_{1} \phi_{2},
$$

for some scalar $\alpha_{1}$. Let

$$
\lambda^{\prime}(s)=\alpha_{0}, \quad u^{\prime}(s)=\alpha_{0} \phi+\alpha_{1} \phi_{2} .
$$

Second differentiation of (2.13) gives, at $s=s_{0}$,

$$
G_{u}^{0} u_{0}^{\prime \prime}(s)=-\left[G_{u u}^{0}\left(u_{0}^{\prime}(s), u_{0}^{\prime}(s)\right)+2 G_{u \lambda}^{0}\left(u_{0}^{\prime}(s), \lambda_{0}^{\prime}(s)\right)+G_{\lambda \lambda}^{0}\left(\lambda_{0}^{\prime}(s)\right)^{2}\right]-G_{\lambda}^{0} \lambda_{0}^{\prime \prime}(s) .
$$

The bracketed term is in $R a\left(G_{u}^{0}\right)$ and hence

$$
\int \frac{\partial u_{0}}{\partial x_{n}}[---]=0
$$

This after simplification results in

$$
\begin{aligned}
& a \alpha_{0}^{2}+2 b \alpha_{0} \alpha_{1}+c \alpha_{1}^{2}=0, \\
& a=\int \frac{\partial u_{0}}{\partial x_{n}} G_{u u}^{0}\left(\frac{\partial u_{0}}{\partial x_{n}}, \frac{\partial u_{0}}{\partial x_{n}}\right), \\
& b=\int \frac{\partial u_{0}}{\partial x_{n}} G_{u u}^{0}\left(\frac{\partial u_{0}}{\partial x_{n}}, \phi\right), \\
& c=\int \frac{\partial u_{0}}{\partial x_{n}} G_{u u}^{0}(\phi, \phi) .
\end{aligned}
$$


Equation (2.12) can be rewritten as

$$
\begin{aligned}
-\Delta \phi & =f^{\prime}(u) \phi-1 & \text { in } \Omega, \\
\phi & =0 \quad \text { on } \partial \Omega, & \int_{\Omega} \phi \frac{\partial u_{0}}{\partial x_{n}}=0 .
\end{aligned}
$$

Since there is no radial degeneracy, there exists a unique radial $\phi$ satisfying this equation. This $\phi$ satisfies the orthogonality condition also and hence by uniqueness this must be the solution of (2.14). Thus $a$ and $c$ have to vanish. We now show $b \neq 0$.

The uniqueness arguments given above and equation (2.4) give

$$
\phi(r)=\frac{p-1}{2 p \lambda}\left(r u^{\prime}+\frac{2}{p-1} u\right), \quad \phi^{\prime}(1)=\frac{p-1}{2 p} .
$$

By differentiating equation (2.1),

$$
-\Delta(\Delta u)=f^{\prime \prime}(u)|\nabla u|^{2}+f^{\prime}(u) \Delta u \quad \text { in } \Omega .
$$

Green's formula gives

$$
\int_{\Omega}(\Delta u \cdot \Delta \phi-\phi \Delta(\Delta u))=\int_{\partial \Omega} \frac{\partial \phi}{\partial n} \Delta u-\int_{\partial \Omega} \phi \frac{\partial}{\partial n}(\Delta u),
$$

which after simplification results in

$$
-\lambda \phi^{\prime}(1) \operatorname{vol}(\partial \Omega)=\int_{\Omega} f^{\prime \prime}(u)|\nabla u|^{2} \phi .
$$

(2.15) now shows $b \neq 0$, since at least for some $i$,

$$
\int_{\Omega} f^{\prime \prime}(u)\left(\frac{\partial u}{\partial x_{i}}\right)^{2} \phi \neq 0 .
$$

Without loss of generality, we let $i=n$.

We have at $\left(u_{0}, \lambda_{0}\right)$ :

(i) $G_{u}^{0}$ is singular and dimension $\left(\operatorname{ker} G_{u}^{0}\right)=1 ; \operatorname{codimension}\left(R a G_{u}^{0}\right)=1$.

(ii) $G_{\lambda}^{0} \in R a\left(G_{u}^{0}\right)$.

(iii) The nondegeneracy condition $b \neq 0$ holds.

Now using standard arguments (cf., for example, [1]) it is easy to show that indeed $\left(u_{0}, \lambda_{0}\right)$ is a bifurcation point. The two distinct curves cutting at $\left(u_{0}, \lambda_{0}\right)$ have the tangent directions

$$
\left(d u_{1}, d \lambda_{1}\right)=(1, \phi), \quad\left(d u_{2}, d \lambda_{2}\right)=\left(0, \frac{\partial u_{0}}{\partial x_{n}}\right)
$$

the first being the radial solution branch and the latter being the branch of nonradial solutions.

3. Concluding remarks. After analyzing the proofs more carefully, the following remarks seem in order.

REMARK 1. In one dimension, for the odd nonlinearity $f(u)=u|u|^{p-1}$, a global picture was obtained in [2] by a scaling argument. When $n>1$, this scaling argument works only for $B_{z(\lambda)}$ where $z(\lambda)$ is the first zero of $u(r)$. It fails outside because of the presence of the term $(n-1) u^{\prime} / r$. But we observe that the proof 
of Theorem 1 will go through for the nonlinearity $f(u)+\lambda$, in the annulus $\tilde{\Omega}=$ $B_{1} \backslash B_{a(\lambda)}$, provided $a(\lambda)$, the last zero of $u(r)$ before 1 , tends to 0 as $\lambda$ tends to $\infty$. Thus we can conclude that each $S_{k}^{ \pm}, k \geq 1$, can exist only in $\left[-\lambda_{k}, \tilde{\lambda}_{k}\right]$, where $S_{k}^{ \pm}=\{(u, \lambda)$ : a solution of $(2.1), u$ has $(k-1)$ nodes in $(0,1), u(0)>0($ or $<0)\}$. As in one dimension, we expect the positive solution branch $S_{1}^{+}$to continue as $S_{3}^{+}$, etc., by developing more and more nodes. Each time a new node is developed, we get a solution $(u, \lambda) \in S_{k}^{ \pm}$with $\partial u / \partial n=0$ on $\partial \Omega$. These are potential symmetry breaking points. We conjecture that the radial solution branch has purely radial degeneracy (turning points) and purely nonradial degeneracy (symmetry breaking points) alternatingly.

REMARK 2. Even though this paper discusses a special class of functions, what seems to be interesting is the way we prove the nondegeneracy. Perhaps it is possible to prove the nondegeneracy (or uniqueness) using similar test functions, wherever the time map is used to prove such results. Hopefully this approach may lead to the settling of the conjecture of Smoller and Wasserman [5].

REMARK 3. Recall that in view of Theorem 1, we concluded that the positive solution branch has to encounter a singular point. This is a consequence of Theorem 1 and also the existence of a priori bounds for positive solutions. (See DeFigueiredo, Lions, Nussbaum, A priori estimates and existence of positive solutions of semilinear elliptic equations, J. Math. Pures Appl. 61 (1983)).

REMARK 4 . Since the unique positive solution of

$$
\begin{aligned}
-\Delta u & =u^{p} \quad \text { in } B_{1}, p<(n+2) /(n-2), \\
u & =0 \quad \text { on } \partial B_{1}
\end{aligned}
$$

is nondegenerate, we can get a branch of positive solutions, using implicit function theorem, for $\varepsilon$ in $\left[0, \varepsilon_{0}\right)$, for equation

$$
\begin{aligned}
-\Delta u & =u^{p}+\varepsilon u^{q} \quad \text { in } B_{1}, \\
u & =0 \quad \text { on } \partial B_{1}
\end{aligned}
$$

for any $q$. This fact has already been observed in a more general set up by Rabinowitz for $q>(n+2) /(n-2)$ (Variational methods for nonlinear elliptic eigenvalue problems, Indiana Univ. Math. J. 23 (1974)) and by Brezis and Nirenberg (Positive solutions of nonlinear elliptic equations involving critical Sobolev exponents, Comm. Pure Appl. Math. 36 (1983)) for $q=(n+2) /(n-2)$, through different arguments.

\section{REFERENCES}

1. G. Cerami, Symmetry breaking for a class of semilinear elliptic problems, Nonlinear Anal. 10 (1986), 1-14.

2. B. Gidas, W. M. Ni and L. Nirenberg, Symmetry and related properties via the maximum principle, Comm. Math. Phys. 68 (1979), 209-243.

3. M. Ramaswamy, On the global set of solutions to a nonlinear ODE-theoretical and numerical description, J. Differential Equations 65 (1986), 1-48.

4. J. A. Smoller and A. G. Wasserman, Existence, uniqueness and nondegeneracy of positive solutions of semilinear elliptic equations, Comm. Math. Phys. 95 (1984), 129-159.

5. __ Symmetry breaking for positive solutions of semilinear elliptic equations, Arch. Rational Mech. Anal. 95 (1986), 217-225.

Tata Institute of Fundamental Research, Bangalore, Karnataka State, 560012 INDIA 